\title{
Early Detection of Acute Kidney Injury in Newborns with Respiratory Distress Syndrome by measurement of serum cystatin c
}

G.S.Abdelmotaleb ${ }^{1}$, A.M.Diab ${ }^{1}$, W.A.Abdelhalem ${ }^{2}$ and F.S.Elshaarawy ${ }^{1}$

${ }^{1}$ Pediatrics Dept., Faculty of Medicine, Benha Univ., Benha, Egypt

${ }^{2}$ Clinical Pathology, Dept., Faculty of Medicine, Benha Univ., Benha, Egypt

E-Mail:Fatma26@gmail.com

\begin{abstract}
Acute kidney injury (AKI) is an important contributing factor to the morbidity and mortality of critically ill neonates. Neonates with respiratory distress syndrome (RDS) are at increased risk of AKI due to exposure to hypoxia, acidosis, hypovolemia, vasopressors and mechanical, the aim of the study is to compare serum cystatin C levels (sCysC) in preterm neonates with respiratory distress syndrome (RDS) with a control group and to evaluate the role of serum cystatin $\mathrm{c}$ in prediction of acute kidney injury in preterm with RDS a prospective study (case-control) Conveyed crazy from february 2019 on january 2020. The contemplate might have been led on 62 neonates starting with the neonatal emergency unit units (NICUs) about Benha college healing centers Furthermore Benha protection Hospital, might have been subdivided into; one assembly (I): Which incorporated 34healthy control preterm neonates without RDS ,Group (II): Which included 22 preterm neonates with RDS without AKI Furthermore one assembly (III): Which incorporated 6 preterm neonates with RDS \& AKI, there were six neonates with AKI and 22 neonates without AKI Throughout those 1 st 7 days In view of KDIGO definition about AKI, sCysC levels at 3rd day about life were essentially higher over neonates with RDS Be that as no $\mathrm{AKI}$ and neonates in the control group, serum creatinine ( $\mathrm{sCr}$ ) qualities during 3rd day about an aggregation demonstrated no noteworthy distinction the middle of the individuals with RDS for AKI, RDS What's more no AKI or control groups, $\mathrm{sCysC}$ could be utilized as a free predictor of AKI for preterm neonates with RDS.
\end{abstract}

Keywords: Respiratory distress syndrome, Acute kidney injury, Serum cystatin C .

\section{Introduction}

A standout amongst the The greater part incessant manifestations of respiratory disappointment to preterm neonates may be respiratory misery syndrome (RDS). RDS will be An basic reason for neonatal passing What's more handicap [1], Furthermore very nearly $50 \%$ about neonates with conception weight (BW) under 1,500 g need aid influenced Toward RDS [2]. Notwithstanding late progresses for perinatal and neonatal consideration in RDS counteractive action and treatment, a respectable amount of these neonates fair from intense kidney harm (AKI), Furthermore it may be connected with poor result as a free danger fig $[3$, 4]. Intense renal harm (AKI) brings about diminished renal function, aggregation for nitrogenous waste products, misfortune for liquid offset Also reduction for electrolyte, Furthermore acid-base homeostasis. The frequency of AKI in neonates who were admitted of the neonatal emergency unit units (NICU) ranges from 3. 4\%-71\%. This extensive variety is mostly because of the absence of a universally acknowledged meaning about AKI to premature babies [5-6] those serum creatinine use in the neonatal period is connected with a portion restrictions. Throughout the initial $48-72 \mathrm{~h}$ from claiming life, neonatal serum creatinine still reflects maternal levels Also these qualities might decrease In fluctuating rates In days, relying upon gestational agdistis. Moreover, serum creatinine focuses might not progress until $25-50 \%$ of the kidney work need officially been lost. Serum creatinine varies Eventually Tom's perusing muscle mass, hydration state, period Furthermore sexual orientation [7]. Distinguishing biomarkers, should empower early and touchy identification of AKI, need pulled in those consideration for later Examine. A promptly identification Might give acceptable change done tolerant conclusions [8]. Serum cystatin c's (sCysC) which may be An low atomic impostor protein of the cystatin gang will be a protease inhibitor, generated during An steady rate altogether nucleated cells, uninhibitedly filtrated through the glomerular membrane, totally reabsorbed and corrupted Toward the proximal tubule. SCysC doesn't show up to a chance to be influenced Eventually Tom's perusing particular figure muscle mass, age, gender, incendiary state, alternately dietary states Also doesn't cross the placental obstruction in creatinine [10].

Cystatin c's need every last one of hypothetical properties necessary with make an perfect gas marker from claiming renal capacity. It could a chance to be used to focus renal work to sic Neonates [9]. Serum cystatin c Might recognize AKI sooner over serum creatinine clinched alongside premature neonates with RDS [11]. Those point from claiming this consider might have been will assess part of serum cystatin c's to prediction about AKI Previously, preterm neonates with RDS.

\section{Patients and methods}

This may be a prospective investigation (casecontrol) conveyed crazy starting with february 2019 should january 2020.

The ponder might have been led around 62 preterm neonates the middle of 27 and 32 gestational weeks (GW) from the neonatal emergency unit units (NICUs) for Benha school doctor's facilities Furthermore Benha protection healing facility. Educated composed agrees were taken from folks of the incorporated neonates preceding beginning those examine.

The study gathering might have been subdivided into:. One assembly (I): (control one assembly (Which 
included 34 wellbeing preterm neonates. One assembly (II): (RDS- no AKI (Which included 22 preterm neonates without AKI.

Gathering (III): (RDS-AKI) which incorporated 6 preterm neonates who produced AKI.

Incorporation criteria of patients:. $\bullet$ neonates between 27 and 32. Gestational weeks $(\mathrm{GW})$. • Preterm neonates for analysis from claiming RDS. - sound control for 34 neonates at those same gestational agdistis (GA) What's more sex who were not diagnosed RDS or AKI.

Prohibition criteria of patients:. - Newborns with intrinsic renal anomalies Newborns with intrinsic coronary illness or whatever available huge intrinsic anomalies.

- Neonates for hypoxic ischemic encephalopathy.

- maternal history of (gestational diabetes , chorioamnionitis, urinary tract contamination , medication for nephrotoxic drugs). All contemplated neonates were subjected with full historical backdrop taking, finish clinical examination Also research center evaluation as;. - complete blood count, c's sensitive protein, blood gasses.

- serum cystatin c for day 3 by elisa method.

- Creatinine, blood urea nitrogen Levels will be measured on day 3 \&again looking into day 5, 7 for at incorporated neonates.

Imaging:- midsection $\mathrm{X}$ beam with recognize RDS. Inspecting estimations. Fringe blood tests for biochemical parameters for example, blood urea nitrogen (BUN), Cr, Also sCysC were tackled PND 3. We likewise measured the cr levels,BUN after day 3 On preterm neonates with signs of AKI. Biochemical parameters but sCysC were promptly broke down.

\section{1 Measurable dissection}

Those gathered information were tabulated What's more broke down utilizing SPSS form 16 delicate ware (Spss Inc, Chicago, sick organization). Unmitigated information were exhibited Likewise amount Furthermore percentages, usng chi square test ( X2) What's more Fisher's accurate test (FET) to their dissection. Quantitative information were tried for typicality utilizing Shapiro wilks test, accepting typicality during $\mathrm{P} \geq 0$. 05 . They communicated Concerning illustration mean \pm standard deviation, average Furthermore extend. Typically conveyed information of 2 free gatherings were investigated by learner " $t$ " test, same time non parametric ones were assesses Toward mamoncillo Whitney u test (ZMWU). Krauskal Wallis test (KWT) might have been utilized to non parametric variables "around 3 autonomous aggregations. Matched qualities from claiming babies What's more their moms were tried Toward Wilcoxon test. Non parametric correlations were evaluated Toward Spearman's relationship coefficient ( rho ). Roc bend might have been used to identify cut off worth from claiming serum cystatin c, creatinine, with ideal affectability Furthermore specificity for punctual analysis about AKI. The acknowledged level for noteworthiness in this fill in might have been expressed at 0.05 ( $\mathrm{P}<0.05$ might have been viewed as significant).

\section{Results}

Those examine bunch comprised of 28 preterm neonates with RDS Furthermore 34 solid preterm neonates without RDS Likewise those control bunch. Clinical and biochemical qualities of the investigation number need aid demonstrated Previously, table 1. Of 28 neonates for RDS; six satisfied the criteria to AKI Throughout those To begin with 7 days. Ga Furthermore bw were altogether easier to neonates for RDS over those control one assembly in any case about AKI status ( $\mathrm{p}<0$. 01). Apgar scores during 1 What's more $5 \mathrm{~min}$ were Additionally fundamentally bring down Previously, neonates with RDS contrasted with the control gathering in any case for AKI status ( $\mathrm{p}<0.0001)$. There might have been no distinguished oliguria for any neonates in the control assembly Also neonates with RDS Anyhow no AKI, inasmuch as two neonates with RDS Also AKI formed oliguria; these discoveries were statistically critical (p00. 0001) Table (1).

RDS respiratory distress syndrome, AKI acute kidney injury, $\mathrm{Cr}$ serum creatinine, GFR Schwartz estimated glomerular filtration rate, sCysC serum cystatin C. Data were presented as mean $\pm \mathrm{SD}$ or median (with minimummaximum range) ${ }^{\text {a }}$ Comparison among the RDS-AKI subgroup, RDS-no AKI subgroup and controls (oneway ANOVA test or Kruskal-Wallis test). Asterisks denote significant differences in Tukey's or MannWhitney U test with Bonferroni correction post hoc tests. *; RDS-AKI subgroup or RDS-no AKI subgroup vs. controls .**; RDS-AKI subgroup vs. RDS-no AKI subgroup $^{\mathrm{b}}$ Comparison among the RDS-AKI subgroup, RDS-no AKI subgroup and controls (Chi-square test or Fisher's exact test) ${ }^{c}$ Comparison between RDS-AKI subgroup and RDS-no AKI subgroup (unpaired t test), A $\mathrm{p}$ value less than 0.05 was considered statistically significant, and a $\mathrm{p}$ value less than 0.017 was considered statistically significant after Mann-Whitney U test with the Bonferroni correction was used for multiple comparisons

No significant differences were detected in gender, SGA, and mode of delivery among the groups ( $p>0.05)$. In addition, there were no significant differences in median numbers of intubation at birth, initial $\mathrm{pH}$, mechanical ventilation, and surfactant treatment among groups with RDS with and without AKI ( $p>0.05)$.

The diagnostic performance of $\mathrm{sCysC}$ levels for predicting AKI development was assessed on PND3. sCysC was highly predictive, and the area under the curve (AUC) was 0.89 for predicting the development of AKI within $72 \mathrm{~h}(\mathrm{p}=0.0001)$. we also calculated the cut-off sCysC levels to predict the development of AKI on PND3. At the concentration of $\geq 1.32 \mathrm{mg} / \mathrm{l}$, the sensitivity (\%) and specificity (\%) of sCysC for detecting AKI within $72 \mathrm{~h}$ were 91 and $86 \%$, respectively Fig (1). 
Table (1) Comparing Demographic data, Clinical and biochemical parameters of the preterm neonates with RDS with and without AKI subgroup and control group .

\begin{tabular}{lcccc}
\hline & $\begin{array}{c}\text { Control } \\
\text { group(n=34) }\end{array}$ & $\begin{array}{c}\text { RDS-no AKI } \\
\text { subgroup(n=22) }\end{array}$ & $\begin{array}{c}\text { RDS-AKI } \\
\text { subgroup(n=6) }\end{array}$ & P $^{\mathrm{a}, \mathbf{b}, \mathbf{c}}$ \\
\hline $\begin{array}{l}\text { Gestational age } \\
\text { (weeks) }\end{array}$ & $31.0(28.0-32.0)$ & $29.0(27.0-32.0)^{*}$ & $29.0(28.0-29.0)^{*}$ & $0.0001^{\mathrm{a}}$ \\
Birth weight (g) & $1,511(1,097-1,9)$ & $1207(800-1,920)^{*}$ & $1146(1,020-1,270)^{*}$ & $0.0001^{\mathrm{a}}$ \\
Small for gestational & $0 / 34(0 \%)$ & $2 / 22(9.0 \%)$ & $0 / 6(0 \%)$ & $0.243^{\mathrm{b}}$ \\
age (n, \%) & & & $3 / 6(50.0 \%)$ & $0.969^{\mathrm{b}}$ \\
Male sex (n, \%) & $15 / 34(44.1 \%)$ & $9 / 22(40.9 \%)$ & $1 / 6(16.6 \%)$ & $0.760^{\mathrm{b}}$ \\
Vaginal delivery (n, \%) & $10 / 34(29.4 \%)$ & $5 / 22(22.7 \%)$ & $3.5(3.0-5.0)^{*}$ & $0.0001^{\mathrm{a}}$ \\
Apgar at 1 min & $7.0(5.0-8.0)$ & $4.0(3.0-6.0)^{*}$ & $7.0(6.0-7.0)^{*}$ & $0.0001^{\mathrm{a}}$ \\
Apgar at 5 min & $9.0(7.0-10.0)$ & $5.5(5.0-7.0)^{*}$ & $1 / 6(16.7 \%)$ & $0.0001^{\mathrm{b}}$ \\
Oliguria (n, \%) & $0 / 34(0 \%)$ & $0 / 22(0 \%)$ & $7.06 \pm 0.05$ & $0.161^{\mathrm{c}}$ \\
PH upon admission & Not measured & $7.10 \pm 0.06$ & $6 / 6(100 \%)$ & $1.00^{\mathrm{b}}$ \\
Mechanical ventilation & $0 / 34(0 \%)$ & $19 / 22(86 \%)$ & & $0.370^{\mathrm{a}}$ \\
(n, \%) & & & $17.5 \pm 10.5$ & $0.01^{\mathrm{a}}$ \\
D3 BUN (mg/d) & $12.8 \pm 1.5$ & $13.5 \pm 1.6$ & $19.0 \pm 1.0$ & $0.299^{\mathrm{a}}$ \\
D5 BUN (mg/dl) & $11.2 \pm 2.3$ & $14.2 \pm 1.7$ & $0.97(0.90-1.30)$ & $0.001^{\mathrm{a}}$ \\
D3 Cr (mg/dl) & $0.94(0.55-1.21)$ & $1.0(0.40-1.25)$ & $1.68(1.45-1.9)$ & $0.0001^{\mathrm{a}}$ \\
D5 Cr (mg/dl) & $0.51(0.31-0.81)$ & $0.53(0.23-0.75)$ & $1.59 \pm 0.09 *, * *$ & \\
D3 sCysC (mg/l) & $1.10 \pm 0.17$ & $1.14 \pm 0.1 * * *$ & & \\
\hline
\end{tabular}

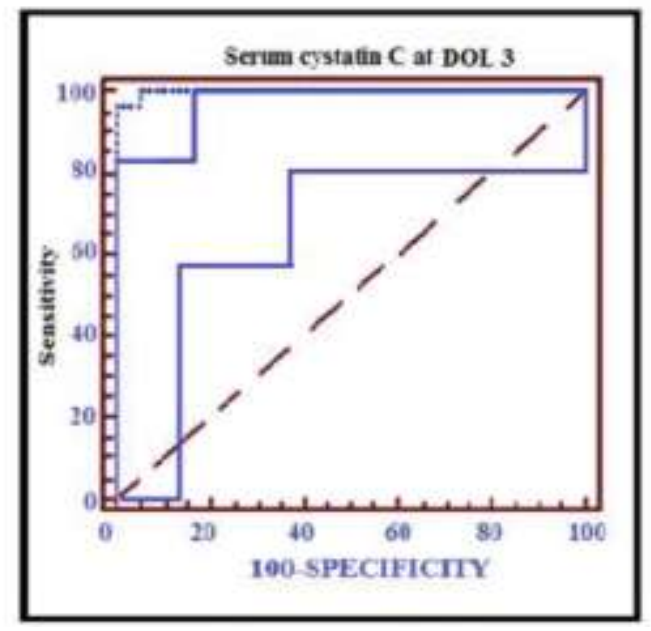

Fig (1) ROC curve for the performance of cystatin c levels in early diagnosis of AKI.

\begin{abstract}
4. Discussion
Our information showed that AKI is portrayed by secondary serum Cystatin c's levels in the RDS-AKI subgroup. Expanded levels for serum Cystatin c's need aid predictive from claiming resulting improvemen from claiming AKI On preterm neonates for RDS. The display investigation looks at the effect from claiming GA, BW, gender, Furthermore Apgar score, on the utility about sCysC Similarly as a AKI biomarker. Regulating for these imperative confounders may be vital done translating AKI biomarkers in the connection of the clinical status from claiming neonates.

Because of inadequate nephrogenesis Furthermore low numbers of nephrons, and additionally extra states for example, such that RDS, preterm neonates would toward danger for AKI [3]. The occurrence of AKI clinched alongside neonates for RDS is showed up for a
\end{abstract}

chance to be in the reach of 25 with $66 \%$ [12]. Previously, our study, the frequency for AKI clinched alongside neonates with RDS might have been $21 \%$, which might have been steady for past investigations.

Our contemplate demonstrated that, there might have been no association the middle of those contemplated aggregations viewing GA, sex What's more mode about conveyance this may be steady for Elmas et al [9]. In neonates, non-oliguric renal disappointment may a chance to be exhibit for more than $50 \%$ of the situations [9]. Best $16.7 \%$ of our patients for AKI required oliguria, and the remaining neonates required non-oliguric AKI.

A low apgar score will be an free hazard variable to impeded renal capacity over preterm neonates for RDS [9, 13, 14]. Over our study, Apgar scores at 1 and 5 min were altogether easier Previously, RDS-AKI neonates 
over neonates with RDS Be that as no AKI Also control Assemblies (P quality $=0.0001)$.

Over Our outcomes we didn't find At whatever huge progress done creatinine levels over neonates with RDS contrasted with controls ahead PND3, Indeed clinched alongside for intensifying renal capacity shown Eventually Tom's perusing oliguria Also expanding serum creatinine. In view serum cystatin c's can't cross those placenta Furthermore stays stable following PND3. To our study, mean sCysC levels were $1.1 \pm 0$. $17 \mathrm{mg} / \mathrm{l}, 1.14 \pm 0.1 \mathrm{mg} / \mathrm{l}$ and $1.59 \pm 0.09 \mathrm{mg} / \mathrm{l}$ for assembly 1 , one assembly 2 and bunch 3 individually their study. Our comes about indicated essentially higher serum cystatin c's focuses in the RDS-AKI subgroup, demonstrating glomerular brokenness looking into PND3. Along these lines we recommended that a expanded sCysC level Might make utilized Likewise an promptly marker about renal capacity to neonates with RDS. The intend qualities for BUN, serum creatinine during the 5th day were altogether higher over AKI bunch over non-AKI aggregation (P value $=0.001)$. Thus $\mathrm{sCysC}$ expands in the blood 1-2 days former should a $50 \%$ Ascent about creatinine. With the goal we discovered that serum cystatin c's Might be utilized Likewise an promptly marker to prediction from claiming AKI.

Th may be hails in understanding with Elmas et al[9], Abdelaal et al[15]they found that serum cystatin c anticipate AKI over preterm for RDS sooner over serum creatinine.

For our study, roc bend dissection indicates that sCysC might have been profoundly predictive, and the territory under those bend (AUC) might have been 0.89 to foreseeing those improvement of AKI inside $72 \mathrm{~h}$ $(p=0$. 0001). We also computed the cut-off sCysC levels to anticipate those advancement of AKI once PND3. In the fixation from claiming $\geq 1.32 \mathrm{mg} / \mathrm{l}$, the affectability (\%) What's more specificity (\%) about sCysC to identifying AKI inside $72 \mathrm{~h}$ were 91 Furthermore $86 \%$, individually. Fig (1).

This will be in line for Abitbol et al [16],Elmas et a [9] who indicated that serum cystatin c's is asuperior biomarker will serum creatinine in the evaluation about GFR Previously, a cross sectional investigation for preterm neonates.

As opposed Cefdar et al[17] who pointed with assess if sCysC Might serve Concerning illustration a exact marker for the analysis about $\mathrm{AKI}$ in the pediatric frigid proclaimed that $\mathrm{sCysC}$ may be a sensitive, Yet not a specific, marker to those finding of AKI On critically sick kids.

\section{Conclusion}

From this study we can demonstrate that serum cystatin c level was found to have a statistically significant association with development of AKI in preterm neonates with RDS and it is increased earlier than serum creatinine which makes it a good predictive marker for AKI in preterm neonates with RDS.

\section{Referencses}

[1] D. Roberts, S. Dalziel , Antenatal corticosteroids for accelerating fetal lung maturation for women at risk of preterm birth. Cochrane Database Syst Rev, vol. 3, PP.CD004454,2006.

[2] J.A. Lemons, C.R. Bauer, W. Oh, et al., Very low birth weight outcomes of the National Institute of Child Health and Human Development Neonatal Research Network, January 1995 through December 1996. NICHD neonatal research network. Pediatrics, vol.107, PP.E1, 2001.

[3] R. Koralkar, N. Ambalavanan, E.B. Levitan, et al ., Acute kidney injury reduces survival in very low birth weight infants. Pediatr Res ., Vol.69,PP.354$358,2006$.

[4] D.J. Askenazi, R. Griffin, G. McGwin,et al ., Acute kidney injury is independently associated with mortality in very low birth weight infants: a matched case-control analysis. Pediatr Nephrol vol.24,PP.991-997, 2009.

[5] P.I. Agras, A. Tarcan, E. Baskin, et al ., Saatci U.Acute renal failure in the neonatal period. Ren Fail, Vol. 26, PP. 305-309, 2004.

[6] C.T. Bezerra, L.C. Vaz Cunha, A.B. Libório, Defining reduced urine Output in neonatal ICU: importance for mortality and acute kidney injury classification. Nephrol Dial Transplant. Vol. 28, PP.901-909, 2013.

[7] Y. Zhang, B. Zhang, D. Wang, et al., Evaluation of Novel Biomarkers for Early Diagnosis of Acute Kidney Injury in Asphyxiated Full-Term Newborns: A Case-Control Study. Medical Principles and Practice . Vol. 30, PP.810-909, 2019.

[8] C.R. Parikh, J.C. Lu, S.G. Coca, Tubular proteinuria in acute kidney injury: a critical evaluation of current status and future promise. Ann Clin Biochem, Vol.47, PP.301-312,2010.

[9] A.T. Elmas, Y. Tabel , O.N. Elmas, Serum CysC predicts acute kidney injury in preterm neonates with respiratory distress syndrome. Pediatr Nephrol, Vol. 28, PP.477-84,2013

[10]M. Treiber, M. Gorenjak, B. Pecovnik Balon, Serum cystatin- $\mathrm{C}$ as a marker of acute kidney injury in the newborn after perinatal hypoxia/asphyxia.TherApher Dial, Vol .18, PP.5767, 2014.

[11] J.G. Jetton , D.J. Askenazi, Update on acute kidney injury in the neonate. Current Opinion in Pediatrics; 24: 191-6,2012.

[12] Y. Li, C. Fu, X. Zhou ,et al., Urine interleukin18and cystatin-C as biomarkers of acute kidney injury in critically ill neonates. Pediatr Nephrol . vol.27, PP.851-860, 2012

[13]L. Cuzzolin, V. Fanos, B. Pinna, , et al., Postnatal renal function in preterm newborns: a role of diseases, drugs and therapeutic interventions. Pediatr Nephrol, Vol.21, PP.931-938 33, 2006.

[14] T. Tulassay, B. Vasarhelyi, Birth weight and renal function. Curr Opin Nephrol Hypertens, Vol.11, PP.347-352, 2002. 
[15] N.A. Abdelaal, S.A. Shalaby, A.K. Khashana, et al., Serum cystatin $\mathrm{C}$ as an earlier predictor of acute kidney injury than serum creatinine in preterm neonates with respiratory distress syndrome. Saudi J Kidney Dis Transpl, Vol. 28(5), PP. 1003-1014, 2017.

[16]C.L. Abitbol, W. Seeherunvong, M.G. Galarza, et al., Neonatal kidney size and function in preterm infants: what is a true estimate of glomerular filtration rate? J Pediatr. Vol.164(5), PP.10261031.e2, 2014 .

[17] O.Y. Safdar, M. Shalaby, N. Khathlan, et al., n Serum cystatin is a useful marker for the ndiagnosis of acute kidney injury in critically ill children: Prospective cohort study. BMC Nephrol, Vol.17, PP.130, 2016. 